\title{
Judith E. TUCKER, Women, Family, and Gender in Islamic
}

\section{Law}

Cambridge, Cambridge University Press, collection "Themes in Islamic Law" 3, 2008, 255 pages

Catherine Mayeur-Jaouen

\section{OpenEdition}

1 Journals

Édition électronique

URL : http://journals.openedition.org/clio/9735

DOI : $10.4000 /$ clio.9735

ISSN : 1777-5299

Éditeur

Belin

Édition imprimée

Date de publication : 1 mai 2010

ISSN : 1252-7017

Référence électronique

Catherine Mayeur-Jaouen, « Judith E. tucker, Women, Family, and Gender in Islamic Law », Clio. Femmes, Genre, Histoire [En ligne], 31 | 2010, mis en ligne le 17 juin 2010, consulté le 21 septembre 2020. URL: http://journals.openedition.org/clio/9735; DOl : https://doi.org/10.4000/clio.9735

Ce document a été généré automatiquement le 21 septembre 2020.

Tous droits réservés 


\title{
Judith E. TUCKER, Women, Family, and Gender in Islamic Law
}

\author{
Cambridge, Cambridge University Press, collection "Themes in Islamic \\ Law" 3, 2008, 255 pages
}

Catherine Mayeur-Jaouen

1 Historienne spécialiste de l'époque ottomane et professeur à l'Université de Georgetown, Judith Tucker fait partie des spécialistes les plus connues de la question du genre au Proche-Orient. Son premier livre, Women in Nineteenth-Century Egypt, 1985, attirait l'attention sur la place des femmes dans le monde du travail dans l'Égypte du XIX ${ }^{e}$ siècle, tandis que le remarquable In the House of Law: Gender and Islamic Law in Ottoman Syria and Palestine, 1998, mettait à jour les résultats du dépouillement des archives des tribunaux et des recueils de fatwas pour les villes de la Palestine ottomane. J. Tucker propose ici une utile synthèse qui vient s'inscrire au côté d'ouvrages collectifs importants parus ces vingt dernières années, comme celui dirigé par Nikki Keddie et Beth Baron (Women in Middle Eastern History, 1991), et de nombreuses monographies de référence (signalons particulièrement Margaret Meriwether, The Kin who Count : Family and Society in Ottoman Aleppo 1770-1840, 1999, et Iris Agmon, Family and Court. Legal Culture and Modernity in Late Ottoman Palestine, 2006, non signalée dans la bibliographie). Le droit musulman est au centre du livre de J. Tucker qui paraît dans une collection spécialisée (Themes in Islamic Law).

2 Le livre est divisé en chapitres thématiques. Après une introduction générale, chacun des chapitres propose sur l'un des quatre thèmes fondamentaux (mariage, divorce, sujet légal, espace public/privé) un rappel des principes de base (Coran, hadîths) qui précède un exposé historique appuyé sur des travaux eux-mêmes basés sur les archives des tribunaux ottomans dans les provinces arabes (surtout aux XVII ${ }^{\mathrm{e}}$ et XVIII ${ }^{\mathrm{e}}$ siècles). J. Tucker propose ensuite un tableau des évolutions les plus récentes, depuis les réformes initiées à la fin du XIXe siècle dans l'Empire ottoman jusqu'aux évolutions les plus récentes du droit de la famille et aux débats actuels (affaires françaises du foulard comprises, mais pas encore le débat sur la burqa'). C'est dans cette dernière partie que J. Tucker évoque le plus les pays musulmans non-arabes, comme la Malaisie et 
l'Indonésie, en rendant compte des campagnes des femmes malaises (notamment de l'association Sisters in islam) pour la revendication de droits dans un cadre de référence scrupuleusement islamique.

3 L'introduction (chapitre I) retrace le développement doctrinal des juristes musulmans et de leur méthodologie, l'histoire des institutions et pratiques légales. Le lecteur profane auquel est destiné le livre pourra ainsi se familiariser avec la Loi islamique dans sa réalité historique, c'est-à-dire le droit musulman et son fonctionnement souple et pluraliste, avec une multiplicité de discours et d'actes légaux où les femmes ont leur place (le droit musulman aussi connaît une female agency). La loi islamique, sans conteste patriarcale dans sa distribution inégale des rôles entre l'homme et la femme, fait en même temps une large place aux expériences proprement féminines, y compris les plus intimes. L'introduction souligne l'effet des réformes des cent dernières années dans le monde musulman, où la rationalisation et l'accroissement du contrôle de l'État ont conduit celui-ci à codifier la loi islamique et à sélectionner un seul avis dans le choix éclectique d'opinions qui prévalait dans les quatre rites juridiques sunnites. Cette standardisation liée à une culture légale plus impersonnelle a pu permettre davantage de protection pour les femmes, au moins en apparence et sur le principe, mais aussi une perte de flexibilité qui est souvent à leur détriment. Les tendances les plus récentes vont à la fois à l'islamisation des systèmes légaux en insistant sur le visible (le voile), mais aussi à une soif normative générale des sociétés musulmanes : jadis adeptes de modèles universels - perçus comme occidentaux - les réformateurs musulmans se basent désormais sur le Coran ou sur de nouvelles interprétations pour réclamer des droits pour les femmes.

Le mariage (chapitre II) est un contrat (nikâh) qui rend les relations sexuelles licites et confère un rôle clair à chacun des époux, le mari pourvoyeur, protecteur et chef du foyer, la femme confinée essentiellement dans des rôles domestiques: le marié doit verser un mahr dont le montant varie, lors du mariage et lors de son éventuelle rupture, puis verser la nafaqa (entretien de l'épouse), tandis que la polygynie est un droit. Des débats juridiques portent sur le mariage des mineures par leurs tuteurs. Mais de nombreux contrats, au moins dans une certaine bourgeoisie urbaine à l'époque ottomane, incluent des stipulations pour protéger la femme et lui conférer certains droits (comme celui de sortir pour aller au bain ou de ne pas avoir de co-épouse). La pensée des réformistes de la fin du XIXe siècle, soucieux de moderniser l'islam et de promouvoir la nation incarnée dans la femme, amena les élites à prôner l'idée de mariage d'amour, à tout le moins le refus du mariage forcé, et à critiquer le mariage précoce. La loi ottomane des droits de la famille en 1917 fixait un âge minimum au mariage ( 9 ans pour les filles, 12 ans pour les garçons), et restreignait le mariage en dessous de 17 ou 18 ans. Dès l'entre-deux-guerres, certains États indépendants comme l'Égypte et la Turquie adoptèrent une législation familiale nouvelle ; pour d'autres pays, ce fut plus tard, lors de la décolonisation (Maghreb) que ces législations furent adoptées : très variées d'un pays à l'autre, "patchworks » juridiques faits de différentes sources et de différentes doctrines (p. 76), les nouvelles lois fixaient en général un âge minimum au mariage, mais évitaient soigneusement certains sujets (nafaqa, mariage civil, héritage). La polygynie ne fut interdite qu'en Turquie et en Tunisie, et seuls certains États soumirent le deuxième mariage à certaines conditions permettant à la première épouse d'être au moins prévenue. Une forte discrimination entre hommes et femmes a finalement persisté avec l'intervention des États et la réforme légale. 
5 Le chapitre sur la répudiation (talâq) porte essentiellement sur l'Empire ottoman, bien qu'il existe aussi des travaux sur la période médiévale. J. Tucker rappelle la tradition légale du talâq, légal mais généralement mal perçu (le Prophète l'avait en horreur), toujours initié par le mari, avec les débats casuistiques sur la 'idda (période qui va de la séparation au divorce final et durant laquelle le mari doit entretenir sa femme) ou sur le droit de la femme de demander le divorce en cas d'absence prolongée du mari. Le khul', procédure où la femme peut demander la séparation (nécessairement avec l'accord du mari) en échange de l'abandon de ses droits financiers, a été très utilisé à l'époque médiévale et ottomane, au moins dans les grandes villes : les réformateurs ont pourtant peu évoqué cette formule qui donne à la femme l'initiative dans la séparation. Ils ont plutôt insisté pour faire passer le talâq au tribunal ou le soumettre à des conditions, mais la loi du divorce est restée «colorée par une vision hautement genrée du pouvoir et de la place de l'homme et de la femme dans le mariage ».

6 Le quatrième chapitre évoque l'homme et la femme comme sujets légaux: le statut marital de la femme n'a pas d'incidence sur sa compétence légale et le mari n'a aucun droit de contrôle sur les biens de sa femme. Mais les femmes n'héritent que de la moitié de la part de leur frère, selon une prescription coranique difficile à réinterpréter, et le témoignage féminin est souvent moins aisément reçu que le témoignage masculin. Cela dit, l'époque ottomane montre le recours important des femmes aux tribunaux pour défendre leurs biens, gérer leur fortune ou établir des fondations pieuses (waqfs).

7 Le cinquième et dernier chapitre traite enfin de la femme et de l'homme dans l'espace public et privé, en rappelant à quel point les débats actuels sur la ségrégation des sexes et sur le voile sont les fruits de la modernité plutôt que l'expression d'une intangible identité islamique : ces débats étaient fort marginaux jusqu'à une date récente, comme le montrent les travaux des historiens. Les ouvrages des juristes islamiques portent finalement sur le contrôle du désir mâle en restreignant - en protégeant - les femmes et leur sortie dans l'espace public: une mixité évidente prévalait pourtant dans les sociétés musulmanes, hier comme aujourd'hui, entre voisins, au marché, lors des mariages ou des pèlerinages, et jusqu'au hajj. La répression de la zinâ' (adultère) montre l'évolution historique (notamment ottomane) qui amena à l'élimination de fait de l'exécution par lapidation comme châtiment de la zinâ'. Quant aux débats des années 1900 sur le hijâb (ségrégation des sexes et port du voile de visage) et le sufûr (dévoilement), ils prônaient l'instruction des femmes et leur sortie pondérée dans l'espace public. Aujourd'hui, note J. Tucker, on assiste à la résurgence (toutefois limitée) du voile de visage au Moyen-Orient sur le modèle saoudien, de même que le châtiment de la zinâ' revient depuis les années 1970 dans la législation de nombreux États (Libye, Pakistan, Soudan, Nigeria) dans des stratégies de légitimation étatique sous couvert d'islamisation.

8 Une courte conclusion, assortie d'un glossaire, d'une solide bibliographie et d'index, pose finalement trois questions. 1) La loi islamique est-elle discriminante pour les femmes? Oui, pour beaucoup de règles relatives au mariage et au divorce, mais la variété des situations prévaut souvent; non, pour la propriété, malgré les règles sur l'héritage. 2) La loi islamique prend-elle en compte la particularité de l'expérience féminine ? Oui, et surtout dans les tribunaux. Mais du coup, la femme est renvoyée à une identité essentialisée qui la marginalise comme sujet. 3) L'homme et la femme comme construction discursives: le rôle féminin est clairement inférieur, mais la femme peut aussi jouer un rôle mâle à l'occasion. J. Tucker conclut finalement au rôle 
ambigu de réformes légales, parfois appauvrissantes, mais qui ont quelquefois permis aux femmes de jouer un rôle sans précédent, comme au Maroc lors de la réforme de la Mudawwana (2004). Au sujet de ces réformes, le lecteur francophone pourra consulter le tout récent volume de Droit et religions, Annuaire, vol. 4, 2009-2010, p. 14-244, publié par l'Université d'Aix-en-Provence, dont une large partie est consacrée aux réformes du droit de la famille dans les sociétés musulmanes.

9 J. Tucker a écrit un ouvrage de synthèse tout à fait accessible aux non-spécialistes ou aux étudiants. On pourra toutefois discuter le titre très général d'un livre qui reste très arabo-centrique : la Turquie et l'Iran sont peu évoqués, le chiisme n'apparaît que pour mémoire, et l'Empire ottoman n'est pas le seul passé des sociétés musulmanes, même s'il est aujourd'hui certainement le mieux connu par l'abondance de ses archives. Des travaux sur l'histoire médiévale auraient pu être également mobilisés. Les mentions faites, ici ou là, de l'Afrique ou de l'Asie (non arabes) sont trop rares. Elles vont d'ailleurs dans le sens général du propos de J. Tucker : elles illustrent la grande variété de situations difficiles à généraliser, où la condition des femmes varie bien autrement que la norme islamique ne le laisse penser. Elles illustrent aussi la tendance actuelle à une sorte de "mondialisation » islamique qui voit éclore partout les mêmes débats sur la place des femmes dans les sociétés musulmanes. 